\title{
Cloning, expression and molecular characterization of a Cystoisospora suis specific uncharacterized merozoite protein
}

\author{
Aruna Shrestha ${ }^{1}$, Nicola Palmieri ${ }^{1}$, Ahmed Abd-Elfattah ${ }^{1}$, Bärbel Ruttkowski ${ }^{1}$, Marc Pagès ${ }^{2}$ and Anja Joachim ${ }^{1 *}$
}

\begin{abstract}
Background: The genome of the apicomplexan parasite Cystoisospora suis (syn. Isospora suis) has recently been sequenced and annotated, opening the possibility for the identification of novel therapeutic targets against cystoisosporosis. It was previously proposed that a $42 \mathrm{kDa}$ uncharacterized merozoite protein, encoded by gene CSUI_005805, might be a relevant vaccine candidate due to its high immunogenic score, high expression level and species-specificity as determined in silico.
\end{abstract}

Methods: The 1170 bp coding sequence of the CSUI_005805 gene was PCR amplified and cloned into the bacterial expression vector PQE-31. The specificity of the expressed recombinant protein was evaluated in an immunoblot, and relative levels of expression in different developmental stages and subcellular localization were determined by quantitative real-time PCR and indirect immunofluorescence assay, respectively.

Results: The CSUI_005805 gene encoded for a 389 amino acid protein containing a histidine-rich region. Quantitative RT-PCR showed that CSUI_005805 was differentially expressed during the early development of C. suis in vitro, with higher transcript levels in merozoites compared to sporozoites. The recombinant protein was specifically recognized by sera from chicken immunized with recombinant CSUI_005805 protein and sera from piglets experimentally infected with C. suis, all of which suggested that despite prokaryotic expression, the recombinant CSUI_005805 protein maintained antigenic determinants and could elicit an immune response in the host. Immunofluorescence labelling and confocal microscopy revealed localization primarily at the surface of the parasite.

Conclusions: The results suggest that CSUI_005805 is highly expressed in merozoites and might thus be critical for their survival and establishment inside host cells. Owing to its specificity, localization and expression pattern, CSUI_ 005805 could be exploited as an attractive candidate for alternative control strategies against $C$. suis such as vaccines.

Keywords: Cystoisosporosis, Recombinant antigen, Invasion inhibition, Protozoa, Swine, Apicomplexa

\section{Background}

Cystoisospora suis (syn. Isospora suis), an enteric protozoan parasite of swine, is a member of the phylum Apicomplexa and the causative agent of neonatal porcine coccidiosis (cystoisosporosis). It is distributed worldwide with high prevalence rates in intensive pig breeding facilities regardless of the farm management system $[1,2]$. Suckling piglets in the first 3 weeks of life are most prone to clinical disease, whereas the infection

\footnotetext{
* Correspondence: Anja.Joachim@vetmeduni.ac.at

'Institute of Parasitology, Department of Pathobiology, University of Veterinary Medicine Vienna, Veterinaerplatz 1, Vienna A-1210, Austria

Full list of author information is available at the end of the article
}

is usually asymptomatic in older piglets with little or no oocyst excretion [3, 4]. Cystoisospora suis has a direct life-cycle with faecal-oral transmission that facilitates its rapid spread among and between litter-mates [5]. Upon ingestion, sporulated oocysts undergo excystation and sporozoites then invade enterocytes to develop into merozoites [6] followed by gamonts [7, 8]. Infected piglets show watery to pasty non-hemorrhagic diarrhea [1], weight loss and uneven weaning weight [9-11] leading to significant economic losses for pig breeders $[12,13]$.

In the European Union, control of cystoisosporosis can currently be accomplished by metaphylactic medication with toltrazuril which is highly effective $[11,12,14$, 
15]. However, emerging resistance is of concern as several incidences of drug resistance against anticoccidials including toltrazuril have already been reported in Eimeria of poultry $[16,17]$, which, along with legislative restrictions on the use of anticoccidials in many countries and increasing consumers' interest in drug residuefree animal products, has led to an urge for development of alternative intervention strategies.

Live, virulent vaccines in large amounts, are impractical for the use in swine as even low infection doses can lead to disease in very young piglets [18], and attenuated lines have not yet been introduced for $C$. suis. An alternative approach would be the introduction of subunit or recombinant vaccines, which demands a systematic search for antigenic proteins to find appropriate vaccine candidates for testing.

The identification of protective antigens is vital for the development of any modern vaccine. In the closely related genus Eimeria, several attempts have been made to identify and characterize antigen-coding transcripts from relevant developmental stages [19-26]. The genome of C. suis has recently been sequenced and contains more than 11,000 protein-coding genes, most of which are expressed in merozoites. However, the functional annotation of coding sequences is still a major challenge. Indeed, more than $40 \%$ of the C. suis genes are currently categorized as of unknown function or annotated as "uncharacterized hypothetical proteins" [27]. The genes with unknown function that are considered unique to $C$. suis may be the most relevant ones to investigate as specific targets for recombinant vaccine development.

In a previous study, $399(34 \%)$ of the 1168 potential vaccine candidates identified by screening of the predicted C. suis proteome also had no annotated function [27]. Homology-based searches indicated that a highly expressed protein of merozoites, encoded by the gene CSUI_005805 and with unknown function, also lacks orthologs in other organisms, making it an attractive target candidate for further research. In the current study, the complete coding region of the CSUI_005805 gene, encoding a novel $C$. suis specific protein, was cloned, expressed in E. coli and characterized in vitro. To date, this is the first attempt to identify and characterize a species-specific antigenic protein of C. suis. Based on immunolocalization and expression pattern of transcripts, it is suggested that this protein may be important for the growth and proliferation of merozoites inside host cells.

\section{Methods}

In vitro culture and parasite harvest

Merozoites of $C$. suis were maintained in intestinal porcine epithelial cells (IPEC-J2) as described elsewhere [28]. Free merozoites were harvested by collecting supernatant of the culture medium 5-6 days postinfection (p.i.), washed with phosphate buffer saline (PBS) and purified using a Percoll density gradient. Further, merozoites were filtered through Partec CellTrics disposable filters $(50 \mu \mathrm{m})$, washed twice with PBS and pelleted by centrifugation at $1000 \times \mathrm{g}$ for $10 \mathrm{~min}$. Purified merozoites were snap frozen in liquid nitrogen and stored at $-80{ }^{\circ} \mathrm{C}$ until further use.

\section{Crude merozoite lysate preparation}

Crude lysate of purified merozoites in PBS was prepared by rapid freeze-thawing using liquid nitrogen followed by disruption in a Tissuelyser II (Qiagen, Hilden, Germany). The preparation was then centrifuged at $20,000 \times \mathrm{g}$ for $10 \mathrm{~min}$ at $4{ }^{\circ} \mathrm{C}$ to separate soluble and insoluble fractions. The insoluble fraction was dissolved separately in buffer with urea (7 M urea, $2 \mathrm{M}$ thiuourea, 4\% 3-((3-cholamidopropyl) dimethylammonio)-1-propanesulfonate, $1 \%(\mathrm{w} / \mathrm{v})$ dithiothreitol, $20 \mathrm{mM}$ Tris). Protein concentration was determined by a Bradford assay [29] using serial dilutions of bovine serum albumin (BSA) as a standard.

\section{Total RNA extraction and CDNA synthesis}

Total RNA was extracted from $4 \times 10^{6}$ purified merozoites using a QIAamp RNA blood mini kit (Qiagen, Hilden, Germany). The RNA preparations were additionally treated with RNase-Free DNase (Qiagen) for $15 \mathrm{~min}$ at room temperature according to the manufacturer's instructions to remove any traces of DNA. Total RNA was quantified using a NanoDrop 2000 (Thermo Fischer Scientific, Waltham, MA, USA) and the integrity was assessed by electrophoresis on a $1 \%$ agarose gel containing ethidium bromide. cDNA was then synthesized from the total RNA using an iScript ${ }^{\text {ts }}$ cDNA synthesis kit (BioRad, Hercules, CA, USA).

\section{Molecular cloning of CSUI_005805 full length CDNA}

The complete coding region of gene CSUI_005805 (1170 bp) (Additional file 1) was obtained by polymerase chain reaction (PCR) amplification using gene-specific primers (forward: 5'-cGA $\boldsymbol{G C T}$ CAA TAC GTC CGG CGT GAA AAT GT-3'; reverse: 5' -gcG TCG ACC TAT AGG AGT TCC ACT AAG GTT-3'). Unique restriction endonuclease recognition sites (bold and italics) were included at the $5^{\prime}$-termini of the primers to facilitate the directional cloning. The target sequence was amplified under the following conditions: an initial denaturation step at $95{ }^{\circ} \mathrm{C}$ for $5 \mathrm{~min}$; followed by 40 cycles of $94{ }^{\circ} \mathrm{C}$ for $15 \mathrm{~s}, 64{ }^{\circ} \mathrm{C}$ for $1 \mathrm{~min}, 72{ }^{\circ} \mathrm{C}$ for $1.5 \mathrm{~min}$ and a final elongation step at $72{ }^{\circ} \mathrm{C}$ for $10 \mathrm{~min}$. Amplification products were loaded onto a $1 \%$ agarose gel stained with ethidium bromide to determine the size of the amplified products. 
The amplicons were gel purified using a QIAquick gel extraction kit (Qiagen), ligated into the pDrive cloning vector (Qiagen), and then used to transform competent Qiagen EZ Escherichia coli cells (Qiagen). The resultant transformants were selected on a Luria-Bertani agar plate supplemented with $100 \mu \mathrm{g} / \mathrm{ml}$ ampicillin (Sigma-Aldrich, St Louis, MO, USA) for resistance and $50 \mu \mathrm{M}$ 5-bromo-4chloro-3-indolyl- $\beta$-D-galactopyranoside (Sigma) and $80 \mu \mathrm{g} /$ $\mathrm{ml}$ isopropyl-1-thio- $\beta$-D-galactopyranoside (IPTG) (Sigma) for blue/white screening. Six recombinant (white) clones were selected and tested by colony PCR using vectorspecific primers. To confirm the integrity of the coding sequences, recombinant plasmids pDRIVE-CSUI_ 005805 were purified using QIAprep spin miniprep kit (Qiagen) and sequenced (Microsynth Austria $\mathrm{GmbH}$, Vienna, Austria) using vector-specific primers.

\section{Sequence analysis of CSUI_005805}

The sequences obtained for the CSUI_005805 cDNA were analyzed for similarity using BLAST programs at NCBI (http://blast.ncbi.nlm.nih.gov/Blast.cgi) and ToxoDB (http://toxodb.org/toxo/showQuestion.do?questionFullName=UniversalQuestions.UnifiedBlast). The deduced amino acid sequence was obtained using the open reading frame (ORF) finder at NCBI (https:// www.ncbi.nlm.nih.gov/orffinder/). The molecular mass, instability index and theoretical isoelectric point were obtained using the ProtParam tool of the ExPASy server of the Swiss Institute of Bioinformatics (http://web.expasy.org/protparam/). Signal peptides, transmembrane regions, subcellular localization and protein motifs were predicted using SignalP (http://www.cbs.dtu.dk/services/ SignalP/), Phobius (http://phobius.sbc.su.se/), PredictProtein (https://www.predictprotein.org/) and Motifscan (http://myhits.isb-sib.ch/cgi-bin/motif_scan) computational tools, respectively.

\section{CSUI_005805 transcription at different time-points of $C$. suis development in vitro}

Real-time quantitative PCR (qPCR) was used to quantify CSUI_005805 transcripts at different time-points of the C. suis development in vitro, namely in free sporozoites released from sporulated oocysts, intracellular merozoites on days 1, 3 and 6 p.i. and extracellular merozoites released into the medium on days 5-6 p.i. Total RNA was extracted from parasites or infected cell cultures at each time-point using an RNeasy mini kit (Qiagen) and treated with RNase-free DNase (Qiagen) to remove any DNA contamination. First-strand cDNA templates were synthesized from $1 \mu \mathrm{g}$ of total RNA using an iScript ${ }^{\mathrm{m}}$ cDNA synthesis kit as described above. Quantitative PCR amplification of cDNA from each time-point of in vitro development was carried out in a Mx3000P thermal cycler (Agilent Technologies, Santa Clara, CA, USA) employing forward and reverse primers $(200 \mathrm{nM}$ each; see Table 1 for details) and $1 \times$ SsoAdvanced $^{\mathrm{Tm}}$ universal SYBR Green supermix (BioRad, Hercules, CA, USA) in a total volume of $20 \mu \mathrm{l}$ using following cycling conditions: initial separation of DNA strands at $95{ }^{\circ} \mathrm{C}$ for $30 \mathrm{~s}$, followed by 40 cycles of $95{ }^{\circ} \mathrm{C}$ for $15 \mathrm{~s}, 60{ }^{\circ} \mathrm{C}$ for $30 \mathrm{~s}$, and $72{ }^{\circ} \mathrm{C}$ for $30 \mathrm{~s}$, and one cycle of $95{ }^{\circ} \mathrm{C}$ for $30 \mathrm{~s}, 60{ }^{\circ} \mathrm{C}$ of $30 \mathrm{~s}$, and $95{ }^{\circ} \mathrm{C}$ for $30 \mathrm{~s}$ for melting-curve analysis. Each sample was run in triplicate and the complete experiment was performed in two separate biological replicates. The qPCR results were normalized against each of the four reference genes, namely glyceraldehyde-3-phosphate, actin, $18 \mathrm{~S}$ ribosomal RNA and large subunit ribosomal RNA genes. Average gene expression relative to the endogenous control for each sample was calculated using the $2^{-\Delta \Delta \mathrm{Ct}}$ method described by Livak \& Schmittgen [30]. Primers for CSUI_005805 gene and four reference genes (Table 1) were designed using Primer3Plus software (http://www.bioinformatics.nl/cgi-bin/ primer3plus/primer3plus.cgi/).

\section{Expression and purification of the recombinant CSUI_005805 protein}

The plasmid pDRIVE-CSUI_005805 with correct sequence and orientation was digested with the restriction enzymes SacI and SalI. The target fragment was then purified, ligated into the expression vector $\mathrm{pQE}-31$ (Qiagen) digested by the same restriction enzymes, and used to transform competent E. coli $\mathrm{M}_{15} \mathrm{pREP}_{4}$ cells (Qiagen) for protein expression. Appropriate target and correct orientation of the inserts were confirmed by colony PCR, restriction analysis and sequencing using $\mathrm{pQE}$ vector specific primers. The recombinant protein expression by $E$. coli clones was induced by adding IPTG (final concentration $1 \mathrm{mM}$ ) after the

Table 1 Oligonucleotide primers used for qPCR to determine stage-dependent transcription of CSUI_005805

\begin{tabular}{llll}
\hline Gene name & Forward primer sequence 5'-3' & Reverse primer sequence 5'- 3' & Amplicon length (bp) \\
\hline GAPDH & ATTGGTCGTCTCGTGTTCCG & GATCGCACTTGGCTCCTTCT & 216 \\
ACT & CTTGCTGGCCGTGATTGAC & ATATTGCCGTCCGGAAGCTC & 203 \\
18S rRNA & GCCAGTAGTCATATGCTTGTC & CTAATAAACACTGCCCTTCCTG & 195 \\
LSU rRNA & TGATTCCGAAGAGTGAGGC & CCAGGCGAAACTATAAAGCAG & 195 \\
CSUI_005805 & CCTGAAAGTCGCCTGTCCAT & GACGCGTCAGCCGTTATAGT & 224
\end{tabular}


$\mathrm{OD}_{600}$ of the culture reached 0.6. The induced bacterial cells were incubated at $37{ }^{\circ} \mathrm{C}$ for $1 \mathrm{~h}$ after adding IPTG and then harvested by centrifugation. Cell pellets were lysed in lysis buffer $\left(100 \mathrm{mM} \mathrm{NaH}_{2} \mathrm{PO}_{4}, 10 \mathrm{mM}\right.$ Tris-Cl, $8 \mathrm{M}$ urea, $\mathrm{pH}$ 8.0) containing ProteoGuard ${ }^{\mathrm{ma}}$ EDTA-Free Protease Inhibitor Cocktail (Clontech, Mountain View, CA, USA) followed by sonication $(65 \%, 60 \mathrm{~s})$ and incubation under constant vortexing for $30 \mathrm{~min}$ at room temperature. Next, the lysate was separated by SDS-PAGE on a $12 \%$ gel and visualized after staining with silver nitrate. The recombinant (His) 6 tagged CSUI_005805 proteins (rCSUI_ 005805) were purified from the soluble fraction of lysates

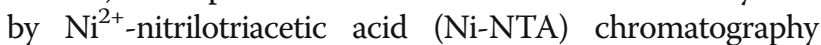
[NiNTA spin columns (Qiagen) for small expression culture and NiNTA agarose (Qiagen) for standard expression culture], as described in the protocols outlined in the QIA expressionist handbook for high-level expression and purification of (His) ${ }_{6}$ tagged proteins (https://www.qiagen.com/ at/resources/resourcedetail?id=79ca2f7d-42fe-4d62-8676-4 cfa948c9435\&lang=en; June 2003). Protein purity was visualized on a $12 \%$ SDS-PAGE gel by silver staining and the concentration was measured by a Bradford assay as described before. The purified protein was stored in aliquots at $-20{ }^{\circ} \mathrm{C}$ until used.

\section{Mass spectrometry}

The protein bands corresponding to rCSUI_005805 were manually excised from silver stained SDS-PAGE gels and subsequently digested in-gel using trypsin (Trypsin Gold, Mass Spectrometry Grade, Mannheim, Germany). The extracted peptides were then subjected to protein identification and quantification using a high-resolution quadrupole time of flight mass spectrometer (Triple TOF 5600+, AB Sciex, Foster City, CA, USA) coupled to a nano-HPLC Ultimate 3000 RSLC system (Dionex, Amsterdam, The Netherlands). The processed MS spectra were searched against UniProt DB (downloaded from the publicly available UniProt server (www.uniprot.org)) together with the in-house generated C. suis database.

\section{Anti-rCSUI_005805 monospecific polyclonal serum production}

Seven-week-old specific pathogen-free (SPF) white leghorn chicken $(n=10)$ were immunized intramuscularly three times at 2-week intervals with purified rCSUI_005805. Primary immunization was performed with $0.1 \mathrm{mg}$ of purified rCSUI_005805 in Freund's complete adjuvant (SigmaAldrich) as a 1:1 emulsion. The birds were boostered twice with the same amount of purified rCSUI_005805 in Freund's incomplete adjuvant (Sigma-Aldrich). Two weeks after the final booster immunization, birds were bled for collection of serum (chicken anti-rCSUI_005805 polyclonal sera). Sera collected before immunization was used as negative control sera (pre-immune chicken sera).

\section{Immunoblot analysis of rCSUI_005805 and crude merozoite lysate}

Crude protein lysate from purified merozoites and rCSUI_005805 was subjected to SDS-PAGE on a $12 \%$ gel and the resolved protein bands were visualized using silver nitrate. The protein bands from the unstained gels were transferred to nitrocellulose (NC) membranes (BioRad) for immunoblot. After blocking for $1 \mathrm{~h}$ with $2 \% \mathrm{BSA}$ in PBS at room temperature, the $\mathrm{NC}$ membranes were incubated with anti-His horseradish peroxidase (HRP) conjugates (Qiagen), chicken anti-rCSUI_005805 polyclonal sera (dilution, 1:200), highly positive porcine anti-C. suis polyclonal sera from experimentally infected piglets (dilution, 1:200) $[31,32]$ or the negative chicken sera (dilution, 1:200) diluted in TTBS buffer $(100 \mathrm{mM}$ Tris, $0.9 \% \mathrm{NaCl}$, $0.1 \%$ Tween 20) for $30 \mathrm{~min}$ at room temperature. To test cross-reactivity of naïve and recombinant antigenic proteins of $C$. suis to Eimeria spp., the NC membranes were incubated separately with field sera obtained from chickens that had been vaccinated with HIPRACOX (HIPRA, Amer, Spain). After rinsing in TTBS for $15 \mathrm{~min}$, blots were exposed to biotinylated goat anti-pig IgG (dilution, 1:400) or biotinylated goat anti-chicken IgY (dilution, 1:300) (Vector Laboratories, Burlingame, CA, USA) diluted in TTBS buffer as secondary antibody for $30 \mathrm{~min}$ at room temperature, incubated with avidin-biotin complex solution (Vector Laboratories) and finally detected by $3,3^{\prime}-5,5^{\prime}$-tetramethylbenzidine, according to the manufacturer's instructions.

\section{Immunofluorescence analysis}

Purified extracellular merozoites and sporozoites were transferred to poly-L-lysine treated glass slides (Polysciences Inc., Hirschberg an der Bergstrasse, Germany) and air dried before fixation. Parasites were either fixed with $4 \%$ paraformaldehyde in PBS for $10 \mathrm{~min}$ followed by permeabilization with $0.25 \%$ TritonX-100 in PBS for $10 \mathrm{~min}$ or fixed in ice-cold $100 \%$ methanol for $10 \mathrm{~min}$ and then blocked with $4 \% \mathrm{BSA}$ in PBS for $2 \mathrm{~h}$ at room temperature. A 1:50 dilution of chicken anti-rCSUI_005805 polyclonal sera was added and incubated for $2 \mathrm{~h}$ at room temperature followed by $1 \mathrm{~h}$ incubation with a 1:300 dilution of Alexa Fluor (A488) goat anti-chicken IgG (Invitrogen, Eugene, OR, USA). Nuclei were visualized by staining with $1 \mu \mathrm{g} / \mathrm{ml}$ of 4,6-diamidino2-phenylindole (DAPI) (Sigma-Aldrich) for $5 \mathrm{~min}$ prior to mounting under coverslips with Aqua-Poly/ Mount (Polysciences Inc.). The slides were washed five times with PBS for $25 \mathrm{~min}$ after each step described above. Imaging was done on a Zeiss Axio Imager Z2 wide-field fluorescence microscope $(\times 63$ oil 
immersion objective) and a Zeiss LSM 510 Meta-confocal laser scanning microscope ( $\times 63$ oil immersion objective). Images were analyzed with Light Editions of Zen 2012 and 2009 (Carl Zeiss Microimaging GmbH, Jena, Germany).

\section{Inhibition of host-cell invasion in vitro}

A qPCR assay was developed in combination with in vitro culture to determine efficacy of the inhibition of host-cell invasion by sporozoites. IPEC-J1 cells $\left(1.5 \times 10^{5}\right.$ cells/well) were seeded in 12-well plates (TPP, Trasadingen, Switzerland) and cultured in Dulbecco's Modified Eagle's Medium (DMEM-Ham's F12) (Gibco, Grand Island, NY, USA) supplemented with $5 \%$ fetal calf serum, $2 \mathrm{mM}$ L-glutamine, $100 \mathrm{U} / \mathrm{ml}$ penicillin and $0.1 \mathrm{mg} / \mathrm{ml}$ streptomycin (Gibco) at $37{ }^{\circ} \mathrm{C}$ with $5 \% \mathrm{CO}_{2}$ for $24 \mathrm{~h}$. Freshly-excysted sporozoites were counted and preincubated at $37{ }^{\circ} \mathrm{C}$ with different concentrations of either chicken anti-rCSUI_005805 or porcine anti-C. suis polyclonal sera for $2 \mathrm{~h}$. The corresponding concentrations of pre-immune chicken serum or previously collected pre-colostral piglet serum [31, 32] were used as a negative controls and equivalent volumes of complete DMEM-Ham's F12 medium as a baseline control. Pre-incubated sporozoites $\left(2 \times 10^{3} /\right.$ well $)$ were used to infect IPEC-J1 cells and the cells were allowed to grow at $40{ }^{\circ} \mathrm{C}$ with $5 \% \mathrm{CO}_{2}$ for $24 \mathrm{~h}$. Cultures were then washed four times with PBS and subjected to DNA extraction using a peqGOLD Microspin Tissue DNA Kit (peqlab, Erlangen, Germany), according to the manufacturer's instructions. The DNA was eluted with $75 \mu \mathrm{l}$ of elution buffer and subjected to qPCR for $C$. suis genome quantification using the large subunit rRNA gene (LSU rRNA gene) as target (GenBank accession number: AF093428.1). qPCR was performed in a Mx3000P thermal cycler (Agilent Technologies, Santa Clara, CA, USA) using $400 \mathrm{nM}$ of each primer (forward: 5'-TGA TTC CGA AGA GTG AGG C -3'; reverse: 5'-CCA GGC GAA ACT ATA AAG CAG -3'), 200 nM probe (5' - FAM-TCC GGC ATT GAT CCC TCT GCT TTA TCC C-BHQ1-3') and $5 \mu \mathrm{l}$ undiluted template DNA with SsoAdvanced ${ }^{\mathrm{m}}$ Universal Probes Supermix (BioRad). Each sample was run in triplicate and the experiment was performed twice under the following cycling conditions: $95{ }^{\circ} \mathrm{C}$ for $10 \mathrm{~min}$ followed by 40 cycles with $95{ }^{\circ} \mathrm{C}$ for $30 \mathrm{~s}$ and $60{ }^{\circ} \mathrm{C}$ for $1 \mathrm{~min}$. In vitro inhibition percentage for each culture was calculated as follows:
The differences among experimental groups were tested by one-way ANOVA using Microsoft Excel 2007, with significance reported at $P<0.05$.

\section{Results}

\section{Cloning and sequence analysis}

The CSUI_005805 gene included a 302 bp 5'-untranslated region (5'-UTR) before the ATG initiation codon and a $1170 \mathrm{bp}$ coding sequence terminating with the TAG stop codon (Fig. 1), followed by a 44 bp 3 '-UTR. A single 1170 bp CSUI_005805 ORF encoded a protein of 389 amino acids with the predicted molecular mass of $42 \mathrm{kDa}$. The theoretical isoelectric point and instability index were 5.52 and 47.77 , respectively. The deduced amino acid sequence had a predicted N-terminal 19amino acids signal peptide (1-26) for entrance into the secretory pathway, and it also had two predicted transmembrane domains (TM1:91-109; TM2:152-171) in the C-terminal region of the protein that could serve as membrane anchorage (Fig. 2). However, it had no identifiable homology to known proteins that might allude to its function. Motifscan predicted two putative glycosylation sites and four putative protein kinase $\mathrm{C}$ phosphorylation sites (Fig. 1).

\section{Real-time quantitative PCR analysis of CSUI_005805 transcripts}

The expression profile of the CSUI_005805 mRNA was examined at different time-points of $C$. suis development in vitro using $\mathrm{qPCR}$. Among the extracellular stages, CSUI_005805 transcripts in merozoites were 18-fold higher (Fig. 3a) compared to sporozoites, which agrees with the high expression level in merozoites measured from an RNA-Seq experiment [27]. Additionally, the level of CSUI_005805 transcripts on day 3 and 6 p.i. were 12- and 21-fold higher, respectively, in intracellular merozoites compared to that of day 1 p.i. (Fig. $3 \mathrm{~b}$ ).

\section{Expression and purification of rCSUI_005805}

The recombinant CSUI_005805 protein (rCSUI_005805) was expressed in M15 pREP4 E. coli as a His-tagged fusion protein. A PCR product of $1170 \mathrm{bp}$ was purified from an agarose gel and cloned into a pre-linearized pQE-31 bacterial expression vector containing $\mathrm{N}$ terminal (His) ${ }_{6}$ tag prior to transformation of chemically competent E. coli M15 pREP4 cells. rCSUI_005805 expressed in bacterial lysate revealed a major protein band of $42 \mathrm{kDa}$ after induction with $1 \mathrm{mM}$ IPTG for

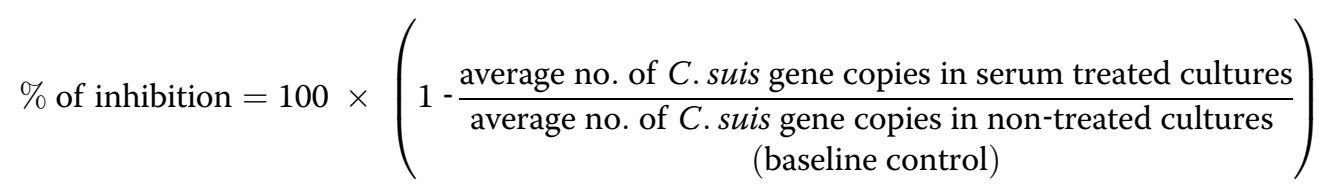




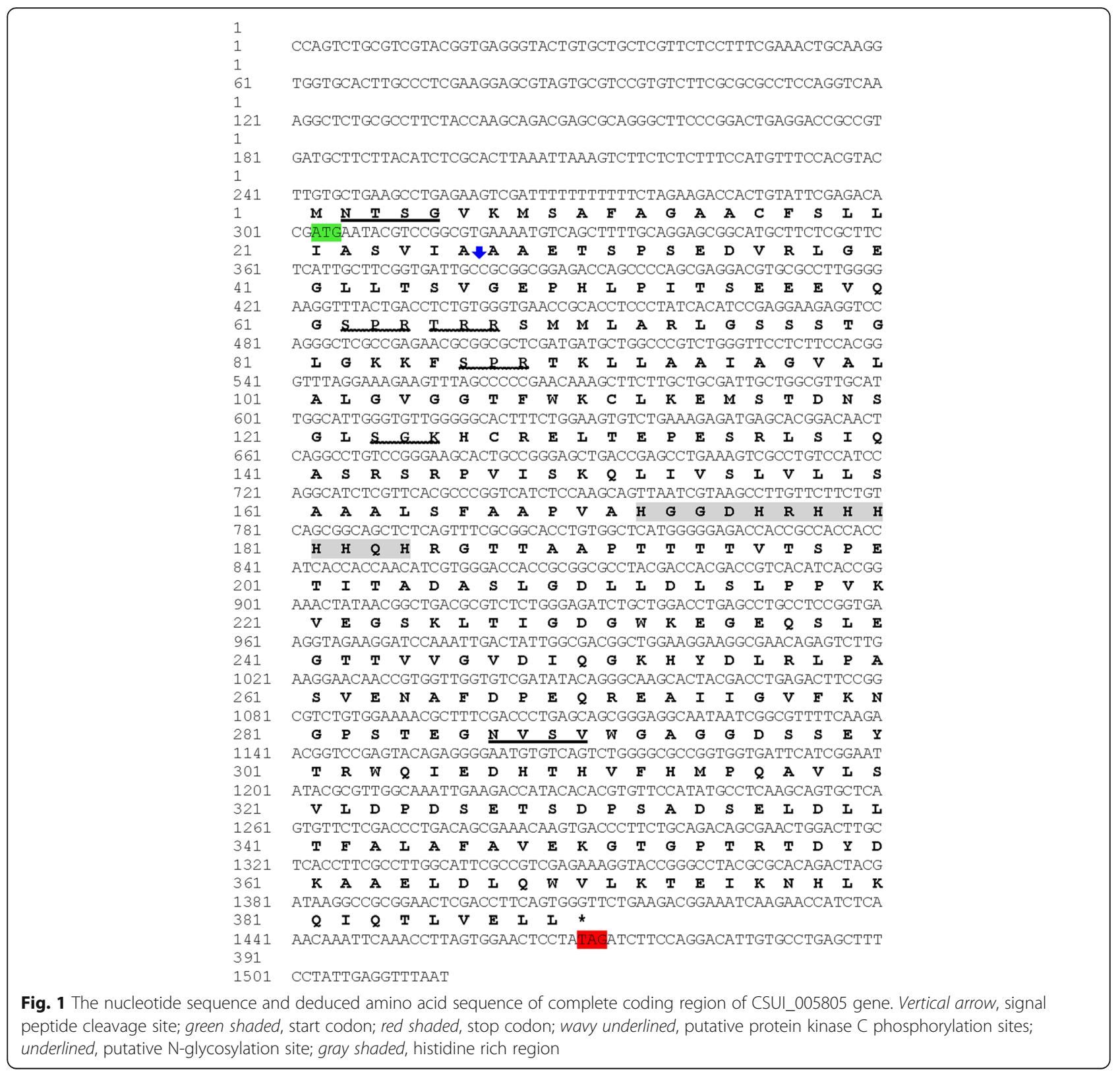

$1 \mathrm{~h}$ at $37^{\circ} \mathrm{C}$ indicating that this was the most appropriate incubation time for expression (Fig. 4a, Lane 3). A band of the target protein was not detected in the bacterial culture that had not been induced with IPTG (Fig. 4a, Lane 1). To isolate any tagged proteins independent of their solubility and location within the cells, purification of rCSUI_005805 was performed under denaturing conditions which allows solubilization of most proteins and inclusion bodies [33], thereby facilitating their direct analysis by SDS-PAGE.

Purification of small expression cultures $(10 \mathrm{ml})$ with NiNTA spin columns after induction with IPTG showed a major protein band of $\sim 42 \mathrm{kDa}$ together with a smaller protein band of $\sim 25 \mathrm{kDa}$ (Fig. 4b, Lanes 1, 2). However, when the expression was scaled up to $500 \mathrm{ml}$ and was batch purified using NiNTA agarose with step-wise elution in elution buffer $\left(100 \mathrm{mM} \mathrm{NaH}_{2} \mathrm{PO}_{4}, 10 \mathrm{mM}\right.$ Tris-Cl, $8 \mathrm{M}$ urea, $\mathrm{pH}$ 4.5), SDS-PAGE showed a major protein band of $\sim 25 \mathrm{kDa}$ and only a faint band of $\sim 42 \mathrm{kDa}$ (Fig. 4C, Lanes 1-5). LC-MS/MS analysis of the $25 \mathrm{kDa}$ band corroborated the presence of a part of the CSUI_005805-coded protein as deduced by nucleic acid translation. Additionally, as (His) ${ }_{6}$ tagged protein, the expression and characterization of the rCSUI_005805 was verified by immunoblot analysis with an anti-His HRP conjugate (Qiagen), reacting specifically as expected (Fig. 4d). 


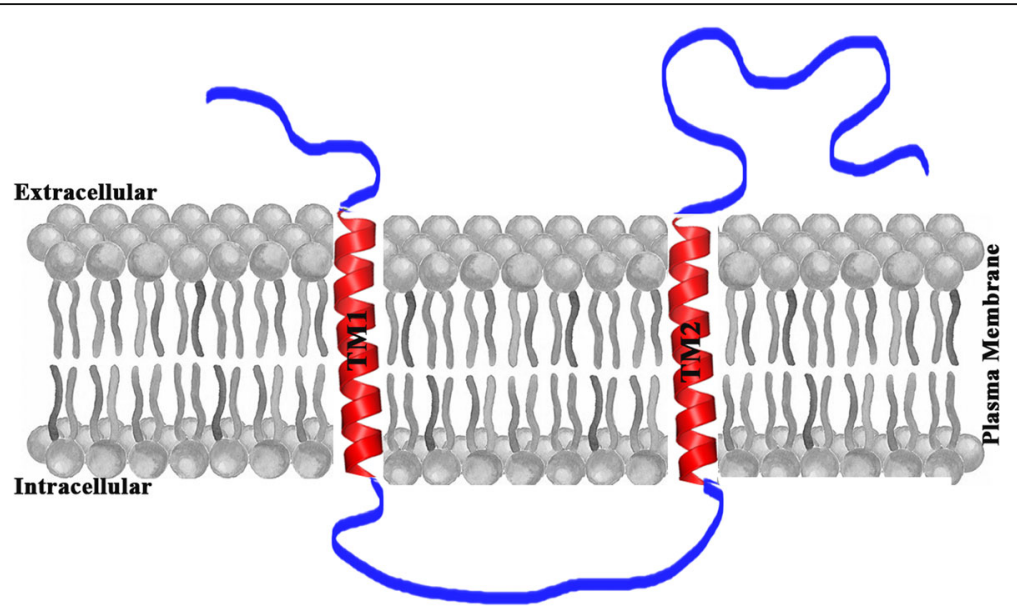

Fig. 2 Schematic representation of predicted transmembrane topology of CSUI_005805 protein. It consists of two transmembrane domains (TM) with extracellular $\mathrm{N}$ and $\mathrm{C}$ termini and a long cytoplasmic loop extending between TM1 andTM2

\section{Immunoblot analysis of rCSUI_005805 and crude} merozoite lysate

The immunoblot of purified rCSUI_005805 probed with highly positive porcine anti-C. suis polyclonal serum could detect the expected $\sim 25 \mathrm{kDa}$ and $\sim 42 \mathrm{kDa}$ protein bands indicating that bacterially expressed rCSUI_ 005805 maintained the antigenic determinants recognized by protective porcine antibodies compared to their naïve protein counterparts (Fig. 5a, Lane 1). Apart from these two bands, a lower molecular weight protein band $(\sim 23 \mathrm{kDa})$ was also detected by porcine anti-C. suis polyclonal sera, which might represent either (a) degraded products, (b) N-terminal proteolytic truncations of rCSUI_005805, since it was only detected by porcine anti-C. suis antibodies but not by anti-His HRP conjugates that recognize the $\mathrm{N}$-terminal poly-histidine tag on recombinant proteins or (c) an unrelated antigenic protein co-purified together with rCSUI_005805. These bands were not detected when porcine pre-colostral serum was used (Fig. 5a, Lane 2). Similarly, chicken antirCSUI_005805 polyclonal sera also recognized purified rCSUI_005805 ( 25 kDa) by immunoblotting (Fig. 5b, Lane 2 ), which confirmed the specificity of the polyclonal sera produced. No bands were detected when the immunoblots were probed with negative (pre-immune) chicken sera (Fig. 5b, Lane 1). Furthermore, to confirm that the chicken anti-rCSUI_005805 sera did in fact recognize the native form of CSUI_005805 protein and not a cross-reactive epitope, an analysis was carried out in which crude merozoite extract was probed with anti-rCSUI_005805 serum. Naïve protein of $42 \mathrm{kDa}$ in insoluble (Lanes 1-3) and soluble (Lanes 4-6) fractions of crude merozoite extract were recognized by chicken anti-rCSUI_005805 polyclonal sera (Fig. 5c, Lanes 3, 6) in a pattern like those identified by highly positive porcine anti-C. suis polyclonal sera (Fig. 5c, Lanes 1,4). As expected, negative chicken serum also failed

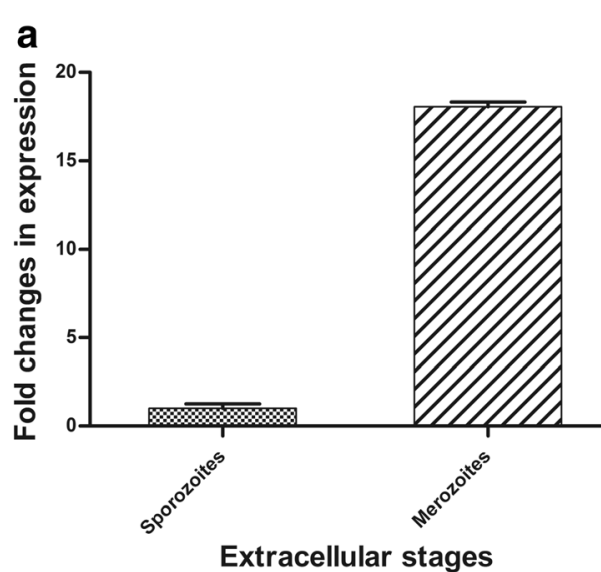

Extracellular stages

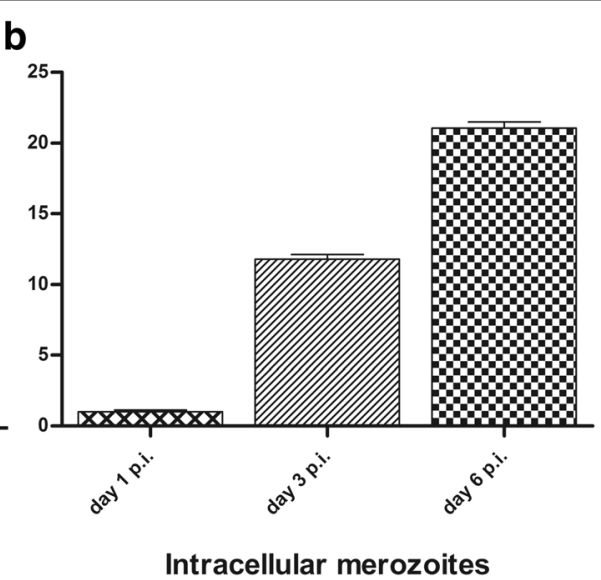

Intracellular merozoites

Fig. 3 Relative mRNA expression levels of CSUI_005805 in vitro. a Free sporozoites and merozoites. $\mathbf{b}$ Intracellular merozoites on day 1, 3 and 6 post-infection (p.i.). The data are displayed as mean \pm standard deviation 


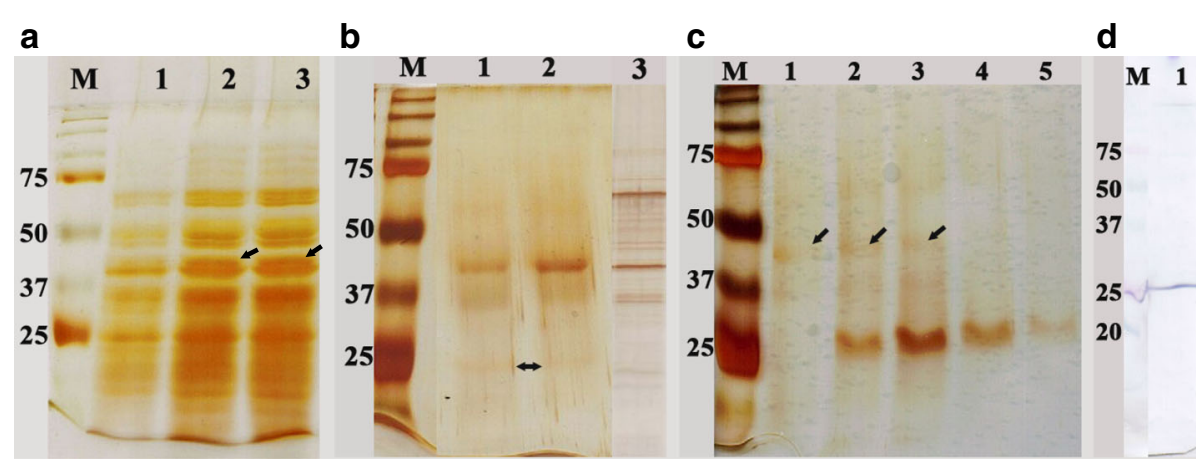

Fig. 4 Expression of rCSUI_005805. Lane M: molecular weight marker. a Analysis of expressed recombinant protein by SDS-PAGE: uninduced culture (Lane 1); induced culture with $1 \mathrm{mM} \mathrm{IPTG}$ for $0.5 \mathrm{~h}$ (Lane 2) and $1 \mathrm{~h}$ (Lane 3), arrows indicate target protein bands of $\sim 42 \mathrm{kDa}$. b SDS-PAGE analysis of NiNTA spin column purified rCSUI_005805: elution 1 (Lane 1); elution 2 (Lane 2), arrows indicate faint protein bands of 25 kDa; naïve merozoite lysate (Lane 3). c Batch purified rCSUI_005805 using NiNTA agarose: stepwise elution (Lanes 1-5), arrows indicate faint protein bands of $\sim 42 \mathrm{kDa}$. $\mathbf{d}$ Immunoblot of batch purified rCSUI_005805 probed with anti-His HRP conjugate (Lane 1)

to detect any bands in an immunoblot of naïve merozoite proteins (Fig. 5c, Lanes 2, 5). None of the antigenic proteins (naïve or rCSUI_005805) of C. suis were recognized by serum antibodies from chickens vaccinated with HIPRA$\mathrm{COX}^{\circ}$ in an immunoblot (Fig. $5 \mathrm{~d}$ ), indicating that C. suis does not share common epitopes with avian Eimeria spp. that are detectable by an immunoblot analysis.

\section{Immunolocalization of CSUI_005805 antigens in merozoites and sporozoites}

Fixed extracellular merozoites and sporozoites were immune-stained with chicken anti-rCSUI_005805 polyclonal serum, visualized with A488 (green) and counter-stained with DAPI (red). Immunofluorescence based localization studies in merozoites showed that irrespective of the fixation techniques used, the labelled antigen was mainly concentrated towards the surface of merozoites (Fig. 6). In some cases, staining was observed towards surface, except for a small gap at the extreme apical and basal ends of merozoites (Fig. 6e), indicating apparent localization of rCSUI_005805 with the inner membrane complex (IMC) [34]. Moreover, the presence of signal peptide and transmembrane domains suggests that rCSUI_005805

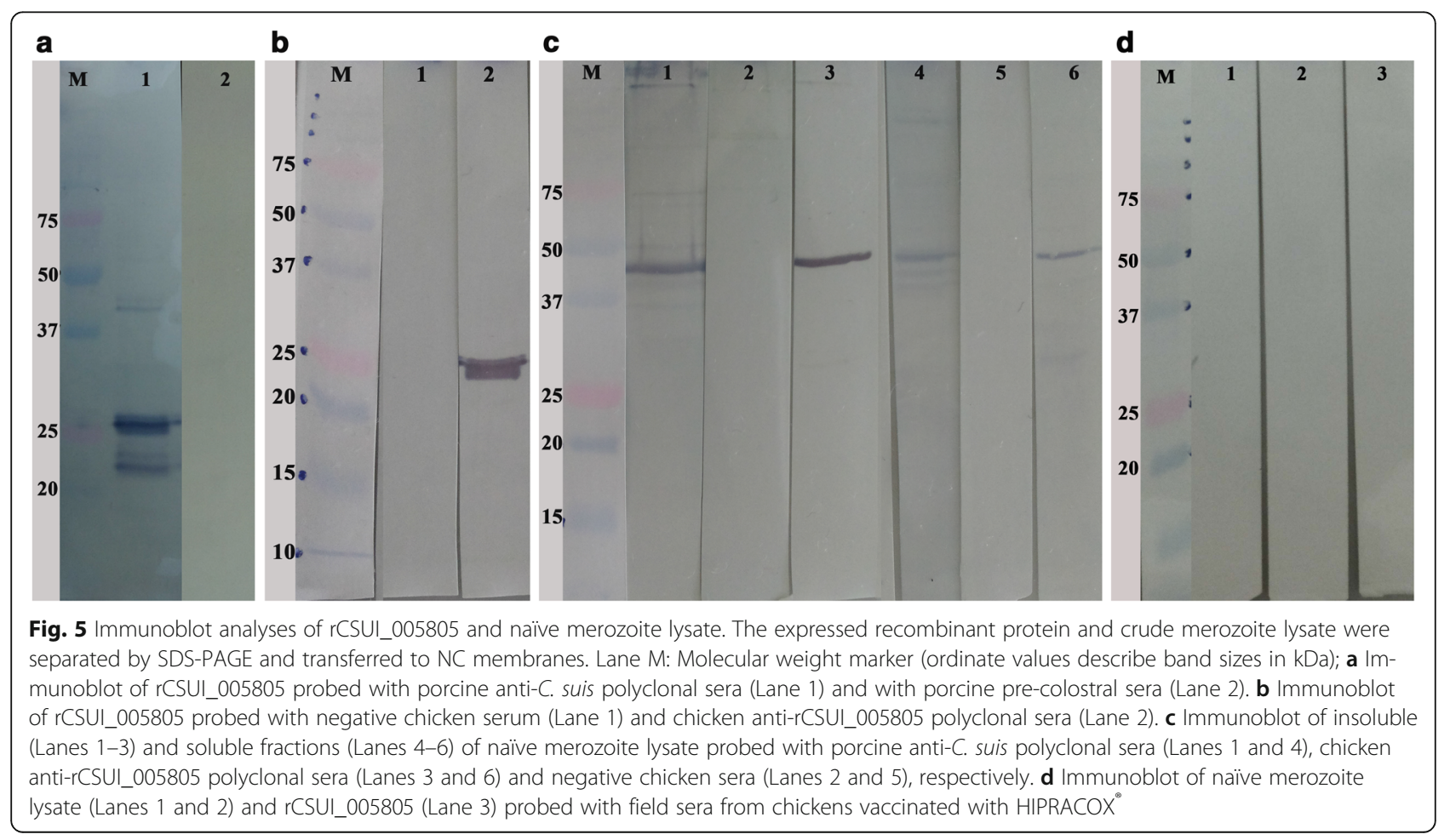




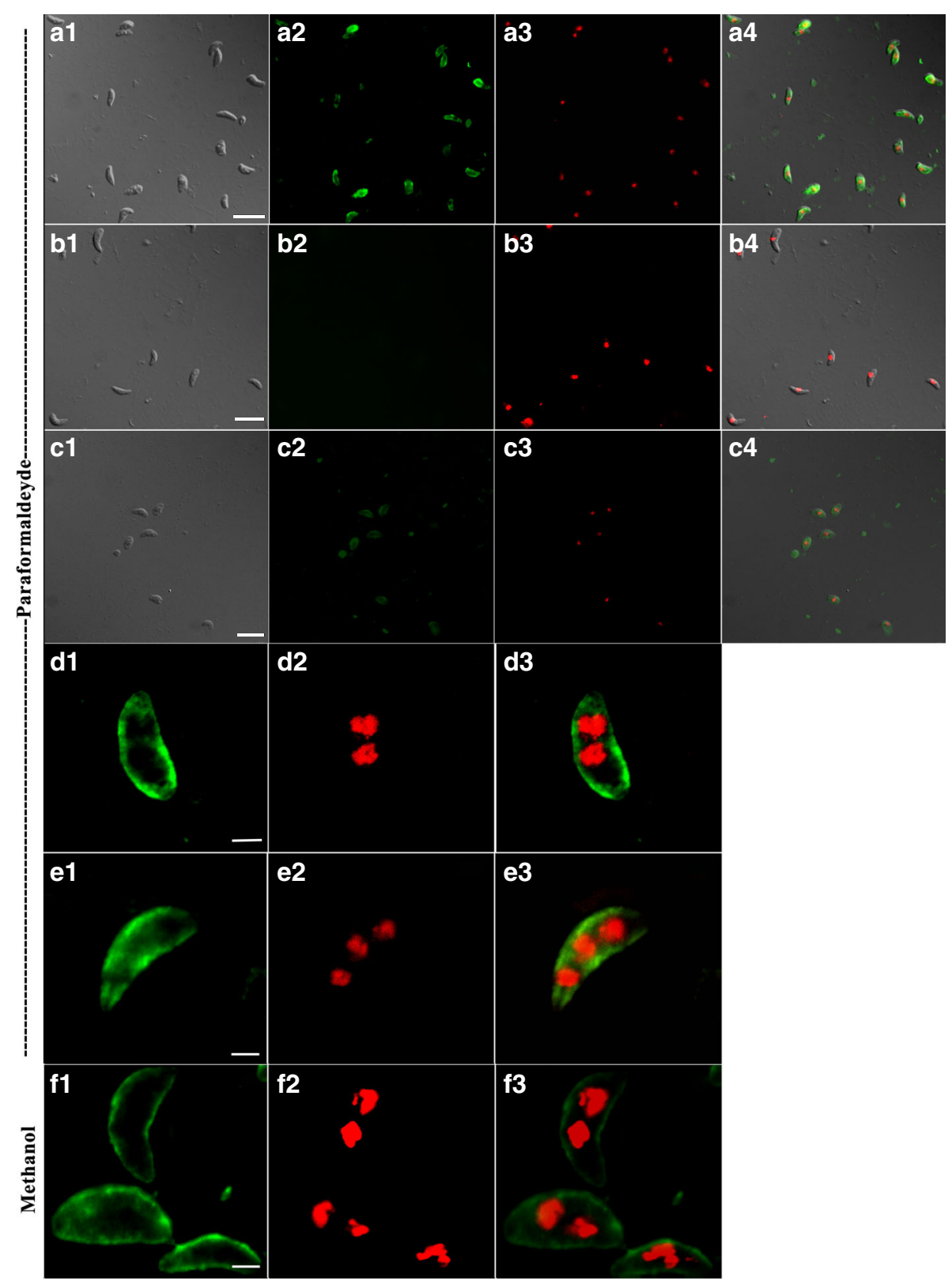

Fig. 6 Localization of CSUI_005805 antigens in merozoites and sporozoites. a-c Wide-field epifluorescence microscope (x63 magnification; scalebar: $10 \mu \mathrm{m})$. $\mathbf{d}-\mathbf{f}$ Meta confocal laser scanning microscope (×63 magnification; scale-bar: $5 \mu \mathrm{m})$. a C. suis merozoites: (a1) Differential interference contrast (DIC); (a2) Localization using A488; (a3) Nuclear staining with DAPI (a4) DIC, A488 and DAPI merged. b Negative control, merozoites probed with negative chicken serum: (b1) DIC; (b2) A488; (b3) DAPI; (b4) merged. c C. suis sporozoites: (c1) DIC; (c2) A488; (c3) DAPI; (c4) merged. d and e Paraformaldehyde-fixed C. suis merozoites: (d1, e1) A488; (d2, e2) DAPl; (d3, e3) merged. f Methanol-fixed C. suis merozoites: (f1) A488; (f2) DAPl; (f3) merged

might be synthesized as mature protein in the cytosol and then transported to the external membrane. The intensity of A488 fluorescence was much lower in fixed extracellular sporozoites than that of merozoites (Fig. 6c). No reactivity was observed when merozoites were stained with negative chicken serum as a primary antibody (Fig. 6b).

\section{Serum invasion-inhibition assay}

Porcine anti-C. suis and chicken anti-rCSUI_005805 polyclonal sera were tested for their ability to inhibit the invasion of cultured IPEC-J1 cells in vitro by C. suis sporozoites. The highest serum concentration that could be employed was $2.5 \%$ because higher concentrations lead to non-specific and non-reproducible results in the assay 
(details not shown). Pre-treatment with positive serum antibodies decreased the invasion capacity of sporozoites as determined by evaluating the number of parasite stages in culture $24 \mathrm{~h}$ after infection, and the observed inhibition effect was dose-dependent. Positive chicken anti- rCSUI_005805 serum (2.5\%) decreased the number of intracellular stages by $28.3 \%$ compared to the baseline control and by $50.6 \%$ compared to the pre-immune serum of the same concentration. In comparison, 2.5\% positive piglet serum decreased the detected number of intracellular stages by $62.1 \%$ compared to the baseline and by $74.2 \%$ compared to pre-colostral serum of the same concentration. Similarly, $1.25 \%$ positive piglet and chicken sera decreased the number of detected stages by 18.5 and $6.8 \%$, respectively, compared to the baseline control and 46.2 and $15.9 \%$, compared to the respective pre-immune serum. For both chicken and piglet sera, pretreatment with 2.5 or $1.25 \%$ positive sera significantly decreased $\left(F_{(1,10)}=13.15, P=0.004 ; \quad F_{(1,10)}=29.42, P=\right.$ 0.0002 , respectively) the invasion capacity of sporozoites while other concentrations had no inhibiting effects.

\section{Discussion}

Cystoisospora suis is an intracellular apicomplexan parasite that develops entirely in single host [35]. Like in other coccidia, replicating merozoites and gamonts are the most pathogenic stages [32, 36, 37], leading to host cell destruction and thus altering intestinal permeability. The global distribution of cystoisosporosis with high prevalence and limited control options demands innovation strategies for novel alternative control measures. The role of humoral immune responses in terms of conferring protective immunity to coccidial infection is debatable. However, evidences of protection by parasitespecific antibodies, either maternally derived or selfproduced, have been reported in Eimeria [38-41] and recently in C. suis [32]. Protective antibodies might thus be important for the defense against invasive stages, sporozoites and merozoites. Cystoisospora suis merozoites express between 9000 and 10,000 proteins during their development [27], thereby exposing the host to a complex assembly of antigens. Logically, the most reliable and effective antigens for raising protective immunity against $C$. suis would be those that are parasite-specific, highly expressed and associated with invasion and/or progression of pathogenesis in the host.

Proteins of unknown function comprise $30-40 \%$ of the total number of proteins annotated from any genome [42]. In all sequenced coccidian genomes, a large fraction of the predicted genes encodes for proteins with no orthologs and/or unknown function [27, 43-46]. These could constitute a rich reservoir for speciesspecific antigens and thus putative targets for immuneintervention. In line with this trend, previous screening of the predicted proteome of C. suis with the software Vacceed revealed numerous potential vaccine candidates with no annotated function [27]. Among these, CSUI_005805, which encodes for an uncharacterized protein, appeared to be an attractive candidate owing to its species-specificity and high expression in merozoites. In the present study, the entire coding sequence of the CSUI_005805 gene was cloned and characterized. A single CSUI_005805 ORF of 1170 bp encoded for 389 amino acids with a predicted molecular mass of $42 \mathrm{kDa}$ and contained a predicted signal peptide and two predicted transmembrane domains. The presence of signal peptide and transmembrane domains suggest that the protein enters the secretory pathway and is actively transported out of the cell to anyone of the numerous cellular membrane systems [47]. Signal peptides are found both in secreted and most cell surface proteins [48], but proteins with predicted signal peptide in combination with transmembrane domains are considered membrane-spanning [47] and might escape secretion. Thus, it is likely that CSUI_005805 is a secretory protein with a signal peptide and/or one or more transmembrane domains for membrane anchorage [49]. Previous studies suggested that most transmembrane proteins encoded by apicomplexans localize to the external membrane or IMC rather than organellar membranes [50-52]. Also, it has recently been reported that in Plasmodium components of the IMC are surfaceexposed and accessible to antibodies [53], and presumably this might also be the case in C. suis, indicating that these proteins could be good targets when selecting for vaccine candidates.

The gene encoding the complete CSUI_005805 protein was cloned into the bacterial expression vector $\mathrm{pQE}-31$ to express the recombinant protein rCSUI_005805 in a size similar to the predicted molecular mass that was present in extracts of the $E$. coli host as visualized in SDS-PAGE by silver staining. However, batch purification of rCSUI_005805 using NiNTA agarose resulted in a faint $42 \mathrm{kDa}$ and a major $25 \mathrm{kDa}$ protein band, suggesting that rCSUI_005805 either undergoes proteolytic maturation yielding smaller $25 \mathrm{kDa}$ protein fragments or the smaller protein band might be a breakdown product of rCSUI_005805 during affinity purification. Additionally, LC-MS/MS analysis of this smaller protein band was consistent with the amino acid sequence predicted for the CSUI_005805 protein. The observed unstable nature of rCSUI_005805 is also in agreement with the predicted instability index value of 47.77 as proteins with an index $>40$ are considered unstable [54].

During expression and purification, rCSUI_005805 maintained a conformation that was recognized by antiserum raised against naïve proteins in immunoblotting, indicating that despite bacterial expression, rCSUI_005805 
retained antigenic and immunogenic properties and could induce an antibody response in the host. Chicken antirCSUI_005805 antibodies could identify soluble and insoluble fractions of naive merozoite proteins in a pattern similar to that identified by sera of experimentally infected piglets, further suggesting that rCSUI_005805 mimics the antigenic properties elicited by immunization with naïve antigens.

Identification of genes expressed in various stages of the life-cycle of a parasite is critical to understand its developmental biology [55-57] and immunological "bottlenecks" where it is most vulnerable. Relative expression patterns of CSUI_005805 mRNA levels at different cultured stages of $C$. suis showed that CSUI_005805 was differentially expressed with higher transcription in merozoites compared to sporozoites. Once the sporozoites invaded the enterocytes, the transcription level increased steadily throughout the entire merogony, indicating that this protein is important for the survival and establishment of merozoites. Expression of CSUI_005805 transcripts in other stages, especially gamonts, should be investigated to complete the picture.

Chicken anti-rCSUI_005805 polyclonal serum was used to determine the subcellular localization of the CSUI_ 005805 protein in fixed extracellular sporozoites and merozoites. Apart from a slightly diffuse cytoplasmic distribution, a major proportion localized at the surface of the parasite, indicating that the investigated protein might be secreted and then translocated to the external membrane as indicated by the results of in silico analyses outlined above. Fixation techniques with either paraformaldehyde or methanol did not alter the localization pattern of this protein. In sporozoites fluorescence intensity of A488 was lower compared to merozoites with both fixation techniques, which supports the results of qPCR that detected increasing levels of CSUI_005805 transcription during in vitro cultivation of $C$. suis as described above.

The ability of apicomplexan parasites to invade host cells is key to their survival and pathogenesis [58]. Host cell invasion is a complex process and is relatively conserved among the members of this taxon [59]. Genomic DNA extracted from parasites after cultivation for $24 \mathrm{~h}$ can be used as a template for quantitatively assessing invasion [60]. Sporozoites pre-incubated with $2.5 \%$ anti-rCSUI_005805 antibodies prior to infection of IPEC-J1 cells slightly reduced the number of intracellular parasites whereas inhibition of host-cell invasion by porcine anti-C. suis serum antibodies in the same dilution was two-fold higher, indicating that CSUI_005805 does not play a leading role in the invasion of the host-cell, at least not by itself.

\section{Conclusions}

We successfully cloned and characterized a novel gene of C. suis, CSUI_005805, which encodes for a species- specific, differentially expressed transmembrane protein. The recombinant CSUI_005805 protein seemed to undergo proteolytic maturation after purification but retained the antigenic and immunogenic properties and is recognized by host antibodies. In vitro expression of CSUI_005805 transcripts increases steadily as soon as sporozoites invade IPEC-J1 cells in vitro, indicating that it might be crucial for establishment and/or growth of merozoites inside the host cells. Currently, no genetic manipulation technique to confirm the direct implication of CSUI_005805 in parasite development is available for $C$. suis. Nevertheless, we have shown that rCSUI_005805 could explicitly induce an antibody response in infected hosts, and given that humoral immune responses confer at least partial protection in $C$. suis, this protein could be exploited for the development of alternative control strategies, possibly in combination with other complementary antigens.

\section{Additional file}

Additional file 1: Complete coding DNA sequence (CDS) of the CSUI_005805 gene. (DOCX 13 kb)

\begin{abstract}
Abbreviations
(His): 6x histidine; A488: Alexa fluor 488; ACT: Actin; BSA: Bovine serum albumin; DAPI: 4, 6-diamidino-2-phenylindole; DMEM: Dulbecco's modified eagle's medium; GAPDH: Glyceraldehyde-3-phosphate; HRP: Horseradish peroxidase; IgY: Immunoglobulin Y; IMC: Inner membrane complex; IPEC: Intestinal porcine epithelial cells; IPTG: Isopropyl-1-thio- $\beta-D-$ galactopyranoside; LC-MS: Liquid chromatography-mass spectrometry; LSU rRNA: Large subunit ribosomal RNA; NiNTA: $\mathrm{Ni}^{2+}$-nitrilotriacetic acid; OD: Optical density; PBS: Phosphate buffer saline; PCR: Polymerase chain reaction; qPCR: Quantitative real-time polymerase chain reaction; rRNA: Ribosomal RNA; SDS: Sodium dodecyl sulfate; SPF: Specific pathogen free; TM: Transmembrane domain; TTBS: Tris-buffered saline; UTR: Untranslated region
\end{abstract}

\section{Acknowledgements}

We are grateful to the VetCore Facility for Research at the University of Veterinary Medicine Vienna, and in particular Dr. Martin Glösmann and Dr. Ursula Reichart for assisting in confocal microscopy and imaging, and Dr. Karin Hummel and Mag. Katharina Nöbauer for their technical support in mass spectrometric analysis.

Funding

AS received PhD position from the University of Veterinary Medicine Vienna within the framework of the graduate school "Pig and Poultry Medicine".

\section{Availability of data and materials}

All the datasets analyzed during this study are included within the article and in the Additional file 1.

\section{Authors' contributions}

$A J, A S$ and $A A E$ conceived and designed the study. AS and BR performed the experiments. NP and AS carried out the bioinformatics analyses. MP helped in generation of antisera. AS drafted the manuscript. All authors read and approved the final manuscript.

\section{Competing interests}

The authors declare that they have no competing interests.

Consent for publication

Not applicable. 


\section{Ethics approval}

The procedures involving piglets for collection of oocysts were approved by the institutional ethics committee and the national authority according to $\S$ $26 \mathrm{ff}$ of Animal Experiments Act, Tierversuchsgesetz 2012 - TVG 2012 (license number: 68.205/0034-WF/NI/3b/2016). Additionally, procedures involving chickens for the generation of antisera were approved by the Animal Ethics Committee of HIPRA according to the Catalan Government Animal Protection law (study code EID-2016-092).

\section{Author details}

'Institute of Parasitology, Department of Pathobiology, University of Veterinary Medicine Vienna, Veterinaerplatz 1, Vienna A-1210, Austria. ${ }^{2}$ HIPRA, Amer 17170, Spain.

\section{Received: 22 November 2016 Accepted: 26 January 2017} Published online: 07 February 2017

\section{References}

1. Lindsay DS, Current W, Power T. Enteric coccidial infections and coccidiosis in swine. Compend Contin Educ Pract Vet. 1992;14:698-02.

2. Meyer C, Joachim A, Daugschies A. Occurrence of Isospora suis in larger piglet production units and on specialized piglet rearing farms. Vet Parasitol. 1999;82(4):277-84.

3. Worliczek HL, Mundt HC, Ruttkowski B, Joachim A. Age, not infection dose, determines the outcome of Isospora suis infections in suckling piglets. Parasitol Res. 2009:105 Suppl 1:S157-62.

4. Koudela K, Kučerová S. Role of acquired immunity and natural age resistance on course of Isospora suis coccidiosis in nursing piglets. Vet Parasitol. 1999;82(2):93-9.

5. Shrestha A, Abd-Elfattah A, Freudenschuss B, Hinney B, Palmieri N, Ruttkowski B, Joachim A. Cystoisospora suis - a model of mammalian cystoisosporosis. Front Vet Sci. 2015;2:68.

6. Matuschka FR, Heydorn AO. Die Entwicklung von Isospora suis Biester und Murray 1934 (Sporozoa: Coccidia: Eimeriidae) im Schwein. In: Herter K, editor. Zoologische Beiträge. Berlin: Duncker \& Humblot; 1980. p. 405-76.

7. Harleman JH, Meyer RC. Life cycle of Isospora suis in gnotobiotic and conventionalized piglets. Vet Parasitol. 1984:17:27-39.

8. Lindsay DS, Stuart BP, Wheat BE, Ernst JV. Endogenous development of the swine coccidium, Isospora suis Biester 1934. J Parasitol. 1980;66(5):771-9.

9. Lindsay D, Current W, Taylor J. Effects of experimentally induced Isospora suis infection on morbidity, mortality and weight gains in nursing pigs. Am J Vet Res. 1985;46(7):71511-12

10. Mundt HC, Joachim A, Becka M, Daugschies A. Isospora suis: an experimental model for mammalian intestinal coccidiosis. Parasitol Res. 2006;98(2):167-75.

11. Joachim A, Mundt $H$. Efficacy of sulfonamides and Baycox ${ }^{\circledR}$ against Isospora suis in experimental infections of suckling piglets. Parasitol Res. 2011:109(6):1653-9.

12. Kreiner T, Worliczek HL, Tichy A, Joachim A. Influence of toltrazuril treatment on parasitological parameters and health performance of piglets in the field - an Austrian experience. Vet Parasitol. 2011;183(1-2):14-20.

13. Scala A, Demontis F, Varcasia A, Pipia A, Poglayen G, Ferrari N, Genchi M. Toltrazuril and sulphonamide treatment against naturally Isospora suis infected suckling piglets: Is there an actual profit? Vet Parasitol. 2009:163(4):362-5.

14. Mundt HC, Mundt-Wustenberg S, Daugschies A, Joachim A. Efficacy of various anticoccidials against experimental porcine neonatal isosporosis. Parasitol Res. 2007;100(2):401-11.

15. Rypula K, Porowski M, Kaba J, Gorczykowski M, Deniz A. Effect of isosporiasis prevention with toltrazuril on long-term pig performance. Sci World J. 2012;2012:4.

16. Stephan B, Rommel M, Daugschies A, Haberkorn A. Studies of resistance to anticoccidials in Eimeria field isolates and pure Eimeria strains. Vet Parasitol. 1997:69(1):19-29.

17. Chapman HD. Biochemical, genetic and applied aspects of drug resistance in Eimeria parasites of the fowl. Avian Pathol. 1997:26(2):221-44.

18. Christensen JP, Henriksen SA. Shedding of oocysts in piglets experimentally infected with Isospora suis. Acta Vet Scand. 1994:35(2):165-72.

19. Liu D, Cao L, Zhu Y, Deng C, Su S, Xu J, et al. Cloning and characterization of an Eimeria necatrix gene encoding a gametocyte protein and associated with oocyst wall formation. Parasit Vectors. 2014;7(1):1-12.
20. Binger M-H, Hug D, Weber G, Schildknecht E, Hümbelin M, Pasamontes L. Cloning and characterization of a surface antigen of Eimeria tenella merozoites and expression using a recombinant vaccinia virus. Mol Biochem Parasitol. 1993;61(2):179-87.

21. Laurent F, Bourdieu C, Yvoré P, Péry P. Cloning and expression of CDNA encoding an Eimeria acervulina $70 \mathrm{kDa}$ sporozoite protein which is related to the $70 \mathrm{kDa}$ heat-shock protein family. Mol Biochem Parasitol. 1994;66(2):349-52.

22. Belli SI, Ferguson DJP, Katrib M, Slapetova I, Mai K, Slapeta J, et al. Conservation of proteins involved in oocyst wall formation in Eimeria maxima, Eimeria tenella and Eimeria acervulina. Int J Parasitol. 2009; 39(10):1063-70

23. Han $\mathrm{H}$, Dong $\mathrm{H}$, Zhu S, Zhao Q, Jiang L, Wang $Y$, et al. Molecular characterization and analysis of a novel protein disulfide isomerase-like protein of Eimeria tenella. PLoS One. 2014;9(6):e99914.

24. Zhai Q, Huang B, Dong H, Zhao Q, Zhu S, Liang S, et al. Molecular characterization and immune protection of a new conserved hypothetical protein of Eimeria tenella. PLoS One. 2016;11(6):e0157678.

25. Mohana Subramanian B, Sriraman R, Hanumantha Rao N, Raghul J, Thiagarajan D, Srinivasan VA. Cloning, expression and evaluation of the efficacy of a recombinant Eimeria tenella sporozoite antigen in birds. Vaccine. 2008;26(27-28):3489-96.

26. Jang SI, Lillehoj HS, Lee SH, Lee KW, Park MS, Cha S-R, et al. Eimeria maxima recombinant Gam82 gametocyte antigen vaccine protects against coccidiosis and augments humoral and cell-mediated immunity. Vaccine. 2010;28(17):2980-85.

27. Palmieri N, Shrestha A, Ruttkowski B, Beck T, Vogl C, Tomley F, et al. The genome of the protozoan parasite Cystoisospora suis and a reverse vaccinology approach to identify vaccine candidates. Int J Parasitol. 2016. in press.

28. Worliczek HL, Ruttkowski B, Schwarz L, Witter K, Tschulenk W, Joachim A. Isospora suis in an epithelial cell culture system - an in vitro model for sexual development in coccidia. Plos One. 2013;8(7):e69797.

29. Bradford MM. A rapid and sensitive method for the quantitation of microgram quantities of protein utilizing the principle of protein-dye binding. Anal Biochem. 1976;72(1):248-54.

30. Livak K, Schmittgen T. Analysis of relative gene expression data using realtime quantitative PCR and the $2^{-\Delta \Delta C T}$ method. Methods. 2001:25(4):402-8.

31. Schwarz L, Joachim A, Worliczek HL. Transfer of Cystoisospora suis-specific colostral antibodies and their correlation with the course of neonatal porcine cystoisosporosis. Vet Parasitol. 2013;197(3/4):487-97.

32. Schwarz L, Worliczek HL, Winkler M, Joachim A. Superinfection of sows with Cystoisospora suis ante partum leads to a milder course of cystoisosporosis in suckling piglets. Vet Parasitol. 2014;204(3-4):158-68.

33. Schlager B, Straessle A, Hafen E. Use of anionic denaturing detergents to purify insoluble proteins after overexpression. BMC Biotechnol. 2012;12(1):1-7.

34. Chen A, Kim E, Toh J, Vashisht A, Rashoff A, Van C, et al. Novel components of the Toxoplasma inner membrane complex revealed by BiolD. MBio. 2015; 6(1):e02357-02314.

35. Lindsay D, Dubey J, Blagburn B. Biology of Isospora spp. from humans, nonhuman primates, and domestic animals. Clin Microbiol Rev. 1997;10:19-34

36. Niestrath M, Takla M, Joachim A, Daugschies A. The role of Isospora suis as a pathogen in conventional piglet production in Germany. J Vet Med B Infect Dis Vet Public Health. 2002;49(4):176-80.

37. Soldati D, Foth B, Cowman A. Molecular and functional aspects of parasite invasion. Trends Parasitol. 2004;20(12):567-74.

38. Wallach M, Halabi A, Pillemer G, Sar-Shalom O, Mencher D, Gilad M, et al. Maternal immunization with gametocyte antigens as a means of providing protective immunity against Eimeria maxima in chickens. Infect Immun. 1992;60(5):2036-9.

39. Smith NC, Wallach M, Miller CM, Morgenstern R, Braun R, Eckert J. Maternal transmission of immunity to Eimeria maxima: enzyme-linked immunosorbent assay analysis of protective antibodies induced by infection. Infect Immun. 1994;62(4):1348-57.

40. Wallach M. Role of antibody in immunity and control of chicken coccidiosis. Trends Parasitol. 2010;26(8):382-7.

41. Rose M. Immunity to coccidiosis: maternal transfer in Eimeria maxima infections. Parasitology. 1972;65(2):273-82.

42. Bork P. Powers and pitfalls in sequence analysis: the $70 \%$ hurdle. Genome Res. 2000;10(4):398-400, 
43. Xia D, Sanderson S, Jones A, Prieto J, Yates J, Bromley E, et al. The proteome of Toxoplasma gondii: integration with the genome provides novel insights into gene expression and annotation. Genome Biol. 2008;9(7):1-18.

44. Ramaprasad A, Mourier T, Naeem R, Malas T, Moussa E, Panigrahi A, et al. Comprehensive evaluation of Toxoplasma gondii VEG and Neospora caninum LIV genomes with tachyzoite stage transcriptome and proteome defines novel transcript features. PLoS One. 2015;10(4):e0124473.

45. Blazejewski T, Nursimulu N, Pszenny V, Dangoudoubiyam S, Namasivayam S, Chiasson M, et al. Systems-based analysis of the Sarcocystis neurona genome identifies pathways that contribute to a heteroxenous life cycle. MBio. 2015;6(1):e02445-02414.

46. Reid A, Blake D, Ansari H, Billington K, Browne H, Bryant J, et al. Genomic analysis of the causative agents of coccidiosis in domestic chickens. Genome Res. 2014;24(10):1676-85.

47. Uhlén M, Fagerberg L, Hallström BM, Lindskog C, Oksvold P, Mardinoglu A, et al. Tissue-based map of the human proteome. Science. 2015; 347(6220):1260419.

48. Zhang Z, Henzel W. Signal peptide prediction based on analysis of experimentally verified cleavage sites. Protein Sci. 2004;13(10):2819-24

49. Huynh M-H, Carruthers V. A Toxoplasma gondii ortholog of Plasmodium GAMA contributes to parasite attachment and cell invasion. mSphere. 2016;1 (1):e00012-16.

50. Florens L, Washburn M, Raine J, Anthony R, Grainger M, Haynes J, et al. A proteomic view of the Plasmodium falciparum life cycle. Nature. 2002; 419(6906):520-6.

51. Sam-Yellowe T, Florens L, Johnson J, Wang T, Drazba J, Le Roch $K$, et al. A Plasmodium gene family encoding maurer's cleft membrane proteins: structural properties and expression profiling. Genome Res. 2004;14(6):1052-9.

52. Hall N, Karras M, Raine J, Carlton J, Kooij T, Berriman M, et al. A comprehensive survey of the Plasmodium life cycle by genomic, transcriptomic, and proteomic analyses. Science. 2005;307(5706):82-6.

53. Swearingen K, Lindner S, Shi L, Shears M, Harupa A, Hopp C, et al. Interrogating the Plasmodium sporozoite surface: Identification of surfaceexposed proteins and demonstration of glycosylation on CSP and TRAP by mass spectrometry-based proteomics. PLoS Pathog. 2016;12(4):e1005606.

54. Guruprasad K, Reddy B, Pandit M. Correlation between stability of a protein and its dipeptide composition: a novel approach for predicting in vivo stability of a protein from its primary sequence. Protein Eng. 1990;4(2):155-61.

55. Sullivan WJ, Smith AT, Joyce BR. Understanding mechanisms and the role of differentiation in pathogenesis of Toxoplasma gondii - A Review. Mem Inst Oswaldo Cruz. 2009;104(2):155-61.

56. Gobert GN, Moertel L, Brindley PJ, McManus DP. Developmental gene expression profiles of the human pathogen Schistosoma japonicum. BMC Genomics. 2009;10(1):1-19.

57. Li B-W, Wang Z, Rush AC, Mitreva M, Weil GJ. Transcription profiling reveals stage- and function-dependent expression patterns in the filarial nematode Brugia malayi. BMC Genomics. 2012;13(1):1-15.

58. Sharma P, Chitnis CE. Key molecular events during host cell invasion by apicomplexan pathogens. Curr Opin Microbiol. 2013;16(4):432-7.

59. Bargieri DY, Andenmatten N, Lagal V, Thiberge S, Whitelaw JA, Tardieux I, et al. Apical membrane antigen 1 mediates apicomplexan parasite attachment but is dispensable for host cell invasion. Nat Commun. 2013;4:2552.

60. Mital J, Ward G. Current and emerging approaches to studying invasion in Apicomplexan parasites. In: Burleigh B, Soldati-Favre D, editors. Molecular mechanisms of parasite invasion: subcellular biochemistry. New York: Springer New York; 2008. p. 1-32.

\section{Submit your next manuscript to BioMed Central and we will help you at every step:}

- We accept pre-submission inquiries

- Our selector tool helps you to find the most relevant journal

- We provide round the clock customer support

- Convenient online submission

- Thorough peer review

- Inclusion in PubMed and all major indexing services

- Maximum visibility for your research

Submit your manuscript at www.biomedcentral.com/submit
C Biomed Central 